\title{
Comparison of dose distributions calculated by the cyberknife Monte Carlo and ray tracing algorithms for lung tumors: a phantom study
}

\author{
Canan Koksal',a, Ugur Akbas ${ }^{1}$, Murat Okutan¹, Bayram Demir², Ismail Hakki Sarpun³ \\ ${ }^{1}$ Istanbul University, Oncology Institute, Department of Medical Physics, Istanbul, Turkey \\ ${ }^{2}$ Istanbul University, Science Faculty, Department of Physics, Istanbul, Turkey \\ ${ }^{3}$ Afyon Kocatepe University, Science and Art Faculty, Department of Physics, Afyon, Turkey
}

\begin{abstract}
Commercial treatment planning systems with have different dose calculation algorithms have been developed for radiotherapy plans. The Ray Tracing and the Monte Carlo dose calculation algorithms are available for MultiPlan treatment planning system. Many studies indicated that the Monte Carlo algorithm enables the more accurate dose distributions in heterogeneous regions such a lung than the Ray Tracing algorithm. The purpose of this study was to compare the Ray Tracing algorithm with the Monte Carlo algorithm for lung tumors in CyberKnife System. An Alderson Rando anthropomorphic phantom was used for creating CyberKnife treatment plans. The treatment plan was developed using the Ray Tracing algorithm. Then, this plan was recalculated with the Monte Carlo algorithm. EBT3 radiochromic films were put in the phantom to obtain measured dose distributions. The calculated doses were compared with the measured doses. The Monte Carlo algorithm is the more accurate dose calculation method than the Ray Tracing algorithm in nonhomogeneous structures.
\end{abstract}

\section{Introduction}

CyberKnife (Accuray Inc, Sunnyvale, CA, USA) is a frameless stereotactic radiosurgery system which provides to deliver the high doses to the target using a 6 MV linac mounted on a robotic arm in a single or a small number of fractions. In this system, the uncertainties of the target location are reduced by getting X-ray images during treatment. The system automatically tracks, detects, and corrects for tumor and patient movement. For tracking tumor, there are some methods such as bony structure tracking, fiducial tracking, and soft tissue tracking. There are 12 fixed circular collimators $(5 \mathrm{~mm}$ to $60 \mathrm{~mm}$ in diameter) and IRIS variable collimator to shape the beams. IRIS collimator automatically changes the size of the variable aperture [1].

In clinic implementations, the computer based treatment planning systems (TPS) are used to obtain planned dose distributions on patient. Commercial TPS with have different dose calculation algorithms have been developed for ideal treatment plans. The Ray Tracing (RT) and the Monte Carlo (MC) dose calculation algorithms are available for MultiPlan TPS (Accuray Inc, Sunnyvale, CA, USA). The RT algorithm, which is a correction-based algorithm, calculates doses using measured beam data such as the off-center ratio, tissuephantom ratio, and collimator output factor at reference conditions and uses an effective path length for heterogeneity corrections. This calculation method does not compute electron transport. The MC algorithm uses a virtual source which is similar a linac head to simulate each treatment beam interaction with medium. The MC algorithm takes into account the electronic disequilibrium. Many studies indicated that the MC algorithm yields the more accurate dose distributions in heterogeneous regions such a lung than the other dose calculation algorithms. In MultiPlan TPS, the MC algorithm is used with uncertainty levels and its range levels are from $0.1 \%$ to $4 \%$. The less number of photon simulation is performed if the higher uncertainty level is assigned $[2,3]$.

The dose prediction which is generated by dose calculation algorithms is very important for a successful treatment. AAPM recommends that the dose calculation should be kept within $\% 3$ [4]. In this study, it was investigated which algorithm provides the more accurate dose predication using film dosimetry for lung tumor.

\section{Materials and Methods 2.1 Treatment Planning}

An Alderson Rando anthropomorphic phantom was used for creating Cyberknife treatment plans. The phantom's computed tomography (CT) images were acquired with 1 mm slice thickness and transferred to the MultiPlan TPS. The gross tumor volume (GTV) and critical structures were contoured. The planning target volume (PTV) was generated with $5 \mathrm{~mm}$ margin beyond the GTV. The

\footnotetext{
a Corresponding author: author@email.org
} 
treatment plan was developed using the RT algorithm through the sequential optimization process (Fig. 1). The fixed circular collimators were used. Then, the high resolution plan was recalculated using the MC algorithm with same beam parameters (Fig. 2) and the Gaussian smoothing algorithm was used $(\sigma=0.6)$. In addition, $2 \%$ uncertainty level was applied for a reasonable optimization time. The prescription dose was $600 \mathrm{cGy}$ for GTV. The plans were normalized to the isodose line which covers $100 \%$ of the GTV.

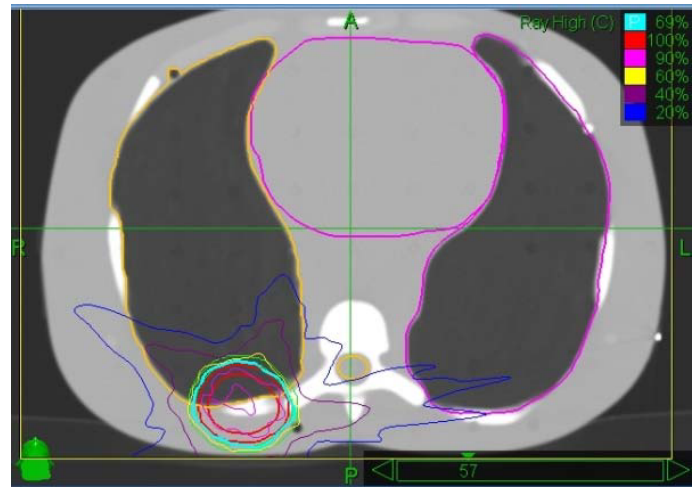

Figure 1. Calculated dose distributions with the RT algorithm

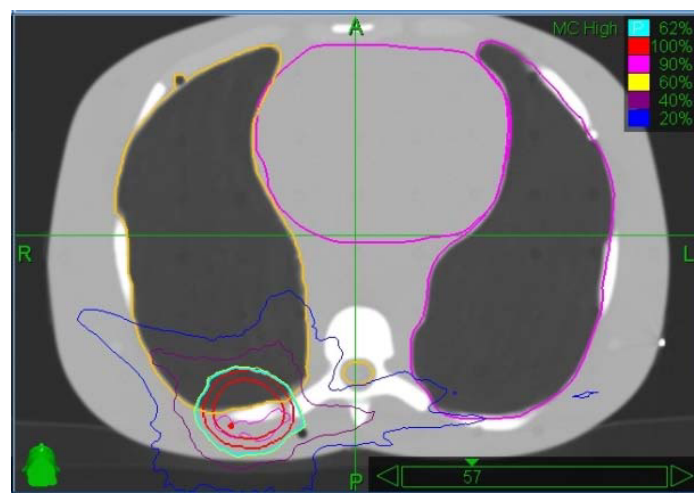

Figure 2. Calculated dose distributions with the $\mathrm{MC}$ algorithm

\subsection{Film Dosimetry}

In this study, the calibration curve was created for film dosimetry. Gafchromic EBT3 (ISP, International Specialty Products, ABD) films were cut in $2 \times 2 \mathrm{~cm}^{2}$ size

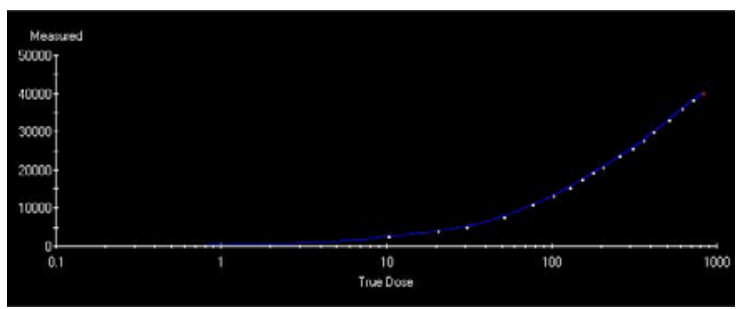

Figure 3. Calibration curve with $6 \mathrm{MV}$ and placed at depth of $5 \mathrm{~cm}$, in a solid water phantom. The source to film distance was $100 \mathrm{~cm}$. Films were oriented perpendicular to the central axis of beam and irradiated with a dose range of $10-800$ cGy using a 6 MV photon energy. The field size was $10 \times 10 \mathrm{~cm}^{2}$ at the isocenter. Films were scanned using a flatbed scanner (Epson 10000XL America Inc., Long Beach, CA) on following day. The optical densities of exposed films were converted to the doses using the Mephysto mcc software program (PTW-New York Corp., Hicksville, $\mathrm{NY}$ ) and the calibration curve was obtained for measurements (Fig. 3).

\subsection{Measurements and Plan Evaluation}

The EBT3 films were put into the phantom to obtain measured dose distributions. The phantom was irradiated using spine tracking method. The reference point doses calculated by TPS and measured point doses with films were compared. There were 4 reference points and their locations were shown in Fig 4. In addition, the treatment plans calculated by the RT and the MC algorithms were evaluated in terms of the target coverage, dose conformity, and dose homogeneity by analyzing the dosevolume histograms. The conformity index (CI) is the ratio of prescription isodose line (PIV) to the tumor volume (TV). CI equal to 1 corresponds to ideal conformation.

$$
\mathrm{CI}=\mathrm{PIV} / \mathrm{TV}
$$

The new conformity index (nCI) formulated by Paddick and expressed as following equation:

$$
\mathrm{nCI}=\left(\mathrm{TV}^{*} \mathrm{PIV}\right) /\left(\mathrm{TV}_{\mathrm{PIV}}\right)^{2}
$$

where $\mathrm{TV}_{\mathrm{PIV}}$ is the target volume covered by the prescription isodose line. Conforming of the prescribed isodose volume to the target shape takes into account in this equation. The homogeneity describes the uniformity of dose within the target volume. The HI formula is:

$$
\mathrm{HI}=\mathrm{D}_{\max } / \mathrm{D}_{\mathrm{Rx}}
$$

where $D_{\max }$ is the maximum dose in the treatment volume and $\mathrm{D}_{\mathrm{Rx}}$ is the prescription dose [5].

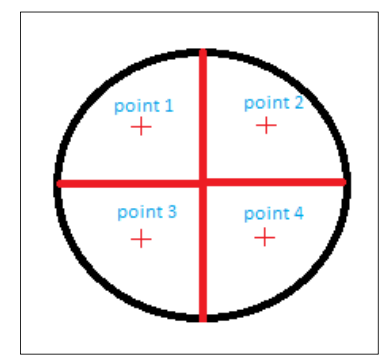

Figure 4. The locations of the reference points in PTV

\section{Results}

The values of reference point doses calculated by the RT and the MC algorithms and measured with EBT3 films 
were shown in Table 1. The differences between the reference point doses calculated by the MC algorithm and measured with films were within $3 \%$. The reference point doses calculated by the RT algorithm were found higher than the $\mathrm{MC}$ algorithm. For point 1, point 2, point 3 , point 4 , the RT algorithm computed doses values of $10.05 \%, 12.35 \%, 11.59 \%$, and $8.26 \%$ greater than the MC algorithm, respectively.

In addition, dose conformity index, homogeneity index, and new conformity index were shown in Table 2 . The RT algorithm produced lower CI and $\mathrm{HI}$ than the MC algorithm. In this study, the prescription isodose line dropped from $69 \%$ for the RT algorithm to $62 \%$ for the MC algorithm.

Table 1. Point doses were calculated by RT and MC algorithms and measured with EBT3 films.

\begin{tabular}{|c|c|c|c|c|}
\hline $\begin{array}{c}\text { Reference } \\
\text { Points }\end{array}$ & RT(TPS) & RT(Film) & MC(TPS) & MC(Film) \\
\hline Point 1 (cGy) & 810 & 746 & 736 & 758 \\
\hline Point 2 (cGy) & 837 & 754 & 745 & 766 \\
\hline Point 3 (cGy) & 828 & 762 & 742 & 764 \\
\hline Point 4 (cGy) & 799 & 741 & 738 & 756 \\
\hline
\end{tabular}

Table 2. Dosimetric parameters

\begin{tabular}{|c|c|c|}
\hline PTV & RT & MC \\
\hline CI & 1.12 & 1.21 \\
\hline $\mathrm{HI}$ & 1.15 & 1.23 \\
\hline $\mathrm{nCI}$ & 1.45 & 1.61 \\
\hline
\end{tabular}

\section{Conclusion}

CyberKnife is a stereotactic radiosurgery, which achieves sparing of critical structures adjacent to the tumors using small fields. This system enables steep dose gradients at the target-normal tissue boundary while delivering the high doses to the target. Therefore, the accurate dose calculation is very crucial in stereotactic radiosurgery.

The dose calculation algorithms are used to predict dose distributions in TPS. In MultiPlan TPS, there are the RT and the MC algorithms. In this study, the calculated doses by the RT and the MC algorithms were compared with the measured doses using film dosimetry for lung tumor. For this investigation, 4 reference points were specified in the target and these point doses were evaluated. The RT algorithm findings were average 10\% higher than the MC algorithm findings.

Wilcox et al. [6] found that the RT algorithm over predicts dose to the PTV and recommended using the MC algorithm for stereotactic radiosurgery of pulmonary targets.

A retrospective study on 33 patients was performed by Vincent et al [7]. The treatment plans were generated using the RT algorithm. Then the plan recalculated with the MC algorithm using the same patient data and treatment parameters. They indicated that the $\mathrm{MC}$ algorithm predicts the more accurate dose distributions than the RT algorithm. The results in this investigation are consistent with literature.

In conclusion, the RT algorithm is overestimated the target doses in heterogeneous medium such a lung. The $\mathrm{MC}$ algorithm predicts the more accurate dose than the RT algorithm because the MC algorithm computes overall photon and electron scatter, particularly heterogeneous medium. However, the MC optimization time is a restrictive parameter in clinics.

\section{References}

1. W. Kilby, J.R Dooley, G. Kuduvalli, S. Sayeh, C.R. Maurer, Technol Cancer Res Treat, 9, 433-452 (2010)

2. F. Crop, Ghent University Faculty of Medicine and Health Sciences, Department of Radiotherapy and Nuclear Medicine, PhD Thesis (2008)

3. S.C. Sharma, J.T. Ott, J.B. Williams, D. DiCKow, J Appl Clin Med Phys, 11, 170-175 (2010)

4. AAPM Report No 54, Woodbury, 22-25 (1995)

5. F. Alejandro, S.O. Iciar, S.R. Alberto, Med Dosim, 39, 1-6 (2014)

6. E. Wilcox, G.M. Daskalov, H. Lincoln, R.C. Shumway, B.M. Kaplan, J.M. Colasanto, Int J Radiat Oncol Biol Phys, 77, 277-84 (2010).

7. W.C. Vincent, T.W. Kwok-wah, T. Shun-ming, J Appl Clin Med Phys,14, 68-78 (2013) 